\title{
Los derechos (no)reproductivos en Argentina: encrucijadas teóricas y políticas
}

\author{
Josefina Leonor Brown **
}

\begin{abstract}
Resumen
El derecho al aborto será la expresión y, en muchos casos, la conquista de una de las demandas feministas centrales en los países del norte durante los años setenta. En los del sur, aquellos fueron los años del sueño de la revolución social y su pulverización bajo una brutal dictadura. Los ecos de aquellas demandas radicales recién pudieron ser escuchadas en Argentina, con el retorno democrático y, con el amparo de los consensos conseguidos en el marco de Naciones Unidas bajo el paraguas de "derechos reproductivos". ¿Por qué esa denominación? De dónde viene y cuáles fueron y son sus posibilidades pero también sus límites en orden a la consideración de las mujeres y otros/otras diferentes como ciudadanas plenas son las preguntas sobre las que pivotearemos.
\end{abstract}

Palavras-chave: Derechos (no)reproductivos, Ciudadanía, Anticoncepción y Aborto, Cuerpo y

Sexualidades, Público y Privado.

\footnotetext{
* Recebido para publicação em agosto de 2006, aceito em julho de 2007. Este trabajo adelanta algunas reflexiones de la investigación para mi tesis doctoral, "Mujeres y ciudadanía en Argentina. Debates teóricos y políticos sobre derechos (no)reproductivos y sexuales (1990-2006)", recientemente defendida en la FSoc/UBA, llevada a cabo con financiamiento del Consejo de Investigaciones Científicas y Tecnológicas (CONICET).

** Doctora en Ciencias Sociales, becaria posdoctoral del CONICET, Universidad Nacional de Buenos Aires (UBA). josefinabrown@gmail.com
}

cadernos pagu (30), janeiro-junho de 2008:269-300. 
Los derechos (no) reproductivos en Argentina

(No)Reproductive Rights in Argentina:

Theoretic and Political Struggle

\begin{abstract}
The right to the abortion will be the expression of one of the most important feminist figth in north countries during the seventies. In those of the south, those were years of the dream of social revolution and of her pulverization under a brutal dictatorship. The echoes of those demands of radical feminists will arrived in the Argentina with the democratic comeback and under protection of the consensuses reached in the frame of United Nations under the umbrella of reproductive rights. What we asked is: why this name, wherefrom does it come and which have been and are his possibilities and his limits in order to the consideration of the women and different others as full citizen?
\end{abstract}

Key Words: (no)Reproductive Rigths, Citizenship, Contraception and Abort, Body and Sexuality, Public-Private. 
El término derechos reproductivos fue objeto de críticas diversas desde que se incorporara al lenguaje común de las demandas de las mujeres y las feministas frente al Estado democrático, a partir de 1983. Desde la popularización del término, las críticas se centraron alrededor de dos ejes. Por un lado, los dardos apuntaban hacia el silenciamiento de la cuestión de la sexualidad y las personas no definidas como heterosexuales. Por otro lado, y lo más criticado sobre todo de parte de las feministas más radicales, fue la expresa referencia a la reproducción y la ausencia explícita de su contracara, la no reproducción (tanto anticoncepción como aborto).

Los cuestionamientos internos (no ya las del Estado y la iglesia) provenían de dos sectores del movimiento: de un lado, de parte de aquellas feministas ligadas a una corriente más radical; de otro, de parte de aquellas y aquellos (feministas y activistas LGTTTBI) que traían a colación los debates que las lesbianas y los y las no heterosexuales estaban acusando a la teoría y la práctica feminista denunciada como blanca $y$ heterosexual. Digamos además que, aún a pesar de lo que la denominación supuestamente englobara (derechos sexuales y aborto), el consenso generado por el término introducido en Naciones Unidas en los ochenta y que aquí aterrizara con fuerza en los noventa, tenía que ver justamente con esas ausencias presencias, sintetizado en "derechos/salud reproductiva". Las relaciones de fuerzas y los consensos internos (muchas veces por presiones externas) hicieron que en la agenda institucional y en las políticas públicas apenas se pueda hablar de salud reproductiva incluso cuando en el último año, el tema del aborto ha podido asomarse a la agenda y ser objeto de algunos debates, ligado precisamente a la salud y la mortalidad de las mujeres en edad reproductiva o en procesos de gestación. El asunto de la libertad ligado a la noción del derecho a decidir sobre los cuerpos, las sexualidades y las capacidades reproductivas queda fuera del paraguas de la salud, despolitizando el asunto y restituyéndolo nuevamente a una decisión privada tomada por un o una experta. 
Los derechos (no) reproductivos en Argentina

¿Por qué entonces derechos reproductivos o derechos sexuales y reproductivos? ¿Se trata de desconocimientos, equívocos o consensos forzados? ¿De dónde viene la noción y cuáles han sido y son sus posibilidades y sus límites en orden a la consideración de las mujeres y otras/os diferentes como ciudadanas/os plenas/os?

\section{Reconstruyendo la historia. El cómo y el por qué de la denominación}

"Derechos Reproductivos o Derechos Sexuales y Reproductivos" o (no)reproductivos, como prefiero llamarlos, son términos polémicos y relativamente recientes que aluden simultáneamente a tres asuntos: 1) los derechos relativos a la seguridad en el embarazo, parto, puerperio pero también acceso a asistencia y tratamientos de fertilización asistida; es decir aquellos eventos relacionados con la reproducción; 2) los derechos relativos a decidir si tener o no hijos o hijas, a decidir con quién, cómo y cuántos y a tener los medios seguros para poder llevar adelante esas elecciones, que se vinculan con anticoncepción y aborto; 3) los derechos relativos al ejercicio libre de la sexualidad sin discriminación, coerción o violencia.

Derechos reproductivos pone el énfasis en el primer aspecto, el reproductivo pero se supone que incluye el segundo: la no reproducción. Para que ambas significaciones se visualicen rápidamente, pongo el (no), entre paréntesis. De allí mi preferencia por la denominación "derechos (no)reproductivos y sexuales".

De cualquier modo, esta terminología es relativamente nueva en el lenguaje de los feminismos. Lo que tiene más historia en el haber de los feminismos y el movimiento de mujeres es la lucha por la autodeterminación y el derecho a decidir sobre el propio cuerpo. Por eso aquí intentaremos rastrear la historia de este concepto, propio de los ochenta (Romero, 2001; Scavone, 1999a, 1999b), y su vinculación con las demandas por el aborto 
Josefina Leonor Brown

libre y legal, la demanda de los sesenta (Gutierrez y Durand, 1998:128; Scavone, 1999a). Se trata de iluminar la trayectoria seguida por los derechos vinculados con la apropiación del cuerpo, el ejercicio libre de la sexualidad, y las capacidades reproductivas. Nos interesa en indagar cuál fue el contexto de emergencia y qué acontecimientos histórico - políticos permiten o producen el pasaje del reclamo por aborto legal hacia un reclamo por derechos reproductivos $\mathrm{o}$, más precisamente salud reproductiva, particularmente, el impacto de esos avatares en la Argentina de los noventa.

\section{El cuerpo como punto de clivaje entre lo público y lo privado}

La llamada primera ola del feminismo tuvo como punto de clivaje de sus demandas la radicalización de la moderna idea de igualdad universal. Para las primeras feministas la equiparación con los varones en el espacio público descansaba en el logro de determinados derechos de los aquellos ya gozaban (voto, trabajo, educación, etcétera). El derecho, espacio de reconocimiento público en los regímenes políticos modernos y consecuentemente, la igualdad de derechos civiles, políticos, culturales, sociales, constituyeron el horizonte de las feministas de la primera ola. Para ellas, la demanda era por igualdad legal sin consideración (o a costa de la abstracción) de la diferencia sexual.

Con la crisis de la modernidad, de la política, de la noción de sujeto que se produce alrededor de los '60, emergerá con nueva fuerza el feminismo en lo que ha sido denominada la segunda ola. Esta irrupción portará algunos cambios significativos con respecto a aquella primera. Por una parte, porque a diferencia de aquella primera oleada ya no será sólo un movimiento de ilustradas y mujeres excepcionales, sino que pasará a ser un movimiento colectivo de características masivas. Por la otra, porque vendrá acompañado de nuevas reivindicaciones. Ya no será sólo cuestión de reducir la diferencia, de salir de lo privado para ingresar al orden público haciendo abstracción de la 
Los derechos (no) reproductivos en Argentina

diferencia sexual. Éste será el momento de cuestionar la base misma de los criterios de distinción y por lo tanto, el acento será puesto en el cuerpo y la sexualidad, como lugares en los que se anuda la diferencia sexual y el dominio patriarcal sobre el género femenino, a partir de la ecuación básica mujer = esposa $=$ madre. Esta crítica vendrá acompañada al mismo tiempo, por el cuestionamiento a la lógica de separación entre lo público y lo privado como lugares exclusivos y privativos de uno u otro sexo, asumiendo el desafío de hacer de lo personal un asunto político.

De ahí que el acento haya sido puesto en la apropiación del cuerpo. El control del cuerpo y la capacidad de las mujeres para decidir sobre el mismo, es visto como el elemento que permitiría la emancipación de las mujeres y otros diferentes. Las feministas de la segunda ola consideraban que la sujeción del cuerpo de las mujeres a complejos mecanismos de prohibiciones - permisiones, era el punto nodal sobre el cual se asentaba el dominio patriarcal. Se trataba de un cuerpo mucho más social que el de los varones, por cuanto el cuerpo de las mujeres resultaba apropiado (o expropiado) en función de su capacidad reproductiva, por el esposo, la Iglesia, el Estado.

Desde los '60, uno de los ejes centrales de la emancipación de las mujeres dentro de los procesos políticos, económicos y sociales, ha estado centrado en torno de la sexualidad. El aborto “...fue crucial en la década del '70 como visualización de la problemática femenina, donde en el derecho al cuerpo se jugarán las percepciones de la diferencia y la igualdad" (Gutierrez y Durand, 1998:128). De lo que se trataba era de separar la sexualidad de la procreación, haciendo de la maternidad una opción y no un destino para las mujeres. Si la capacidad biológica de las mujeres para procrear era uno de elementos centrales para determinar el papel de las mujeres en la sociedad a partir de la maternidad, esto operaría como efecto de totalización semántica mediante la cual, madre es igual a mujer y por deslizamiento de sentido toda mujer es una madre (Rosemberg, 1997:63). Contra esta identificación se alzan las voces feministas, con la pretensión de hacer visibles a las 
mujeres, encubiertas debajo de la imagen maternal. Es decir, de lo que se trataba era de recuperar al sujeto mujer, a las mujeres, independientemente de su potencial capacidad reproductiva.

Entonces, que las mujeres recuperaran su cuerpo, un cuerpo que bajo el mandato de la maternidad obligatoria era un cuerpo para otros, sometido al dominio patriarcal, constituía (y aún sigue constituyendo) un paso fundamental y estratégico para la conquista de la autonomía, la autodeterminación, la libertad de las mujeres; por lo tanto, para remover uno de los puntos centrales en los que se asienta el patriarcado y por ende, la subordinación de las mujeres: el control del cuerpo de las mujeres mediante el control de su sexualidad y sus capacidades (no)reproductivas. De ahí el énfasis puesto y la centralidad adquirida por el reclamo al aborto libre y gratuito, señalando la capacidad de las mujeres de decidir sobre su propio cuerpo y entonces también, como resistencia a aceptar las definiciones hegemónicas vigentes de mujer (Rosemberg, 1997:54). Por otro lado, también, demanda radical para el colectivo de mujeres en una época de cambios y reclamos radicales.

Los años 60-70 fueron tiempos de grandes movimientos sociales, políticos, culturales; momento de cambios y conmociones de muchos de los patrones tradicionales. Época de efervescencia y de grandes utopías, con el movimiento de mujeres en una de sus fases ascendentes. La sexualidad, en este contexto, se planteaba desde el punto de vista de la autodeterminación y la libertad de las mujeres para ejercer una vida sexual plena y ya no, ligada a la reproducción exclusivamente. No existía el VIH-sida y acababa de lanzarse al ruedo la píldora, que prometía liberar de una vez por todas a las mujeres de la función reproductora. La idea de las demandas en los 60/70 giraba en torno de recuperar también para las mujeres, el lado placentero, el del goce en el ejercicio de la sexualidad. Amor libre, revolución sexual, anticonceptivos y aborto libre y gratuito en un mundo donde todo parecía posible, un mundo de "la imaginación al poder". En ese mundo, “(...) El control de la fecundidad era considerado por las pioneras 
Los derechos (no) reproductivos en Argentina

feministas un bien moral - la libertad y la responsabilidad de elegir - del que las mujeres no pueden ser privadas y al que deberían tener acceso sin poner su vida y su salud en peligro" (Gutiérrez y Durand, 1998:158).

Las luchas en EEUU y en las democracias europeas en los años 60/70, estuvieron centradas, por lo tanto, en las demandas por el aborto legal, seguro y gratuito. El tema se planteaba ligado a la lucha por la legalización del aborto y la anticoncepción, en relación con la defensa de la vida de las mujeres. Como sucede hoy en la Argentina, la clandestinidad en la que estas prácticas eran realizadas implicaba serios riesgos para la salud y la vida de las mujeres. Por lo tanto, la legalización y las garantías que le reclamaban al Estado tenían que ver con que no sólo no se realizara fuera de la ley con todos los costos que ello conlleva (económicos, sociales, jurídicos, emocionales y psicológicos), sino que además estuviera al alcance de todas las mujeres y no sólo de quienes, por privilegio de clase, pudieran acceder a estos servicios. $\mathrm{El}$ aborto legal, seguro y gratuito fue una realidad en muchos de los países centrales en aquellos tiempos. Entre los setenta y los inicios de los ochenta Estados Unidos, Alemania, Francia, entre otros, legalizaron el aborto.

Mientras, sobre la curva final de la edad de oro del capitalismo, las demandas de las mujeres y las feministas (como la de tantos otros movimientos sociales) de los países centrales pudieron radicalizarse en ese sentido, en latinoamerica, la radicalización de los setenta adquiría otros ribetes. Esos fueron los años de la revolución social y también los de la brutal dictadura que quebrara ese impulso emancipador.

El cuerpo, las curvas de las mujeres y sus reivindicaciones recién tendrían lugar con el retorno democrático en los ochenta. Para entonces, ya habrían venido la crisis del petróleo y por ende de una redefinición en la economía, concomitante con la crisis de los Estados de Bienestar dando lugar en los '80 a la imposición de un nuevo modelo económico y el establecimiento de nuevas relaciones sociales. Con estos cambios también vendrá el discurso 
neoconservador y con él, el del retorno de la mujer a su rol tradicional (Gutiérrez y Durand, 1998:182).

Así es como frente a las reivindicaciones de las feministas de la segunda ola, para quienes el reclamo por el aborto libre y seguro se basaba en un principio de autonomía mediante el cual ellas recuperaban el derecho a decidir sobre su propio cuerpo, surgirá la reacción de los grupos antiabortistas de la mano de la Nueva Derecha centrados fundamentalmente en torno del llamado "derecho a la vida" (Id. ib.:186). El "derecho a la vida" jugará como un elemento desarticulador del discurso feminista que, de este modo, bajo esta concepción y expuesto en términos dicotómicos, asociará la lucha a favor de la legalización y/o despenalización del aborto con lucha a favor del aborto y, por deslizamiento de sentido, a muerte. De esta manera la discusión quedará entrampada bajo la falsa antinomia: Vida versus Libertad, en la que la libertad de las mujeres a decidir sobre su propio cuerpo es presentada como opuesta al derecho a la vida. La ofensiva fundamentalista católica sobre todo en los países del cono sur, no hará sino incrementarse con el transcurrir de los años. ${ }^{1}$

Todos estos factores, sin dudas, influirán sobre el cambio discursivo operado que implicó la transición desde el reclamo por legalización/despenalización del aborto hacia derechos/salud reproductiva. Las variables expuestas no actuarán solas sino que se conjugarán con otras concurrentes en el tiempo, como la imposición de políticas antinatalistas a determinadas minorías en los países centrales y grandes grupos de mujeres en el sur y el impacto de estos procesos en el movimiento feminista internacional. Todo ello, estará asociado, a su vez, a las

1 Para un panorama más detallado del accionar de la jerarquía católica en los últimos tiempos y la intensificación de su presencia en un lugar privilegiado del espacio público como el actor que presenta mayores resistencia frente a las reivindicaciones de los movimientos de mujeres y las feministas veáse Brown, 2004a:111-126, 2005; Tarducci y Tagliafiero, 2004; Tarducci, 2005; Ciriza, 2004a, $2004 b$. 
Los derechos (no) reproductivos en Argentina

discusiones que se suceden en el plano Internacional en este período, con una creciente participación de las Organizaciones No Gubernamentales (ONG's) vinculadas con las redes feministas y los movimientos de mujeres, en estas discusiones.

Antes de entrar en el impacto de la internacionalización del derecho y la globalización de las desigualdades, vayamos un poco hacia atrás.

Las políticas de población, el neomalthusianismo y los cuerpos de mujeres y sus capacidades reproductivas como objetos de intereses geopolíticos

Entre los años cincuenta y sesenta resurgió la hipótesis malthusiana de explosión demográfica a partir de grupos de investigación, académicos y empresarios británicos y norteamericanos. La atención estaba centrada en el crecimiento poblacional detectado en los países asiáticos y luego en los latinoamericanos. El crecimiento de población en esos países obedecía a dos órdenes de factores. Por un lado, el mantenimiento de la tasa de natalidad en niveles estables; y por otro, el aumento de la esperanza de vida debido a un relativo aumento de la calidad de vida de la población (infraestructura urbana, vacunas, mejoramiento de atención de la salud, control de epidemias, mejoras en la alimentación, etc.) (De Barbieri, 2000:48).

Para frenar este crecimiento poblacional, que era visto como una amenaza a la supervivencia del planeta, los países del sur se vieron sometidos a una serie de presiones para bajar la tasa de natalidad tanto por parte de los organismos internacionales como por parte de los gobiernos de los países centrales. En algunos casos estas presiones internacionales dieron lugar a la imposición de procesos coercitivos de control de la natalidad, como lo ocurrido en la India o Bolivia, por ejemplo. En estos países, se practicaron (en algunos casos aún se siguen practicando) esterilizaciones forzadas y/o la imposición de 
métodos anticonceptivos de larga duración sin información adecuada y/o sin consentimiento de las mujeres. ${ }^{2}$ En ocasiones, estas prácticas han sido usadas como forma de presión para acceder a otro tipo de beneficios sociales $y / 0$ incentivadas mediante premios o recompensas (Sen, Germain, y Chen, 1994).

Esta postura tuvo su contracara en la de los llamados pro natalistas, quienes tampoco estuvieron exentos, en muchos casos, de aplicar métodos coercitivos, aunque no de modo directo sino mediante campañas contra la anticoncepción, la restricción del uso de métodos anticonceptivos a través de trabas legales, y la utilización de distinto tipo de impedimentos para la práctica del aborto, por otra parte penalizado en la mayor parte de los países latinoamericanos. De todas maneras, lo cierto es que, tanto en uno como en otro caso, las mujeres son consideradas como objetos y no sujetos activos y protagónicos de las políticas de población, cuando es justamente su cuerpo el que está en juego en todos los casos. De allí la insistencia creciente del emergente movimiento por la salud y los derechos reproductivos de las mujeres que presionaba para que las mujeres fueran consideran sujetas autónomas capaces de tomar decisiones y no simples objetos o variables de políticas de población.

Los debates, planteados en términos geopolíticos que consideraban a las mujeres, su fecundidad y sus cuerpos como variables del desarrollo económico, parecían no tener salida. La antítesis entre pro y anti natalistas $\mathrm{y} / \mathrm{o}$, entre alternativas de desarrollo periféricas o centrales, encontró su principio de resolución cuando se lo introdujo dentro del paradigma de los derechos humanos, como derechos de las parejas y las familias. De esta manera, el tema de los derechos reproductivos es incluido en la Conferencia de Internacional de Derechos Humanos de Teherán en 1968, en la que se acordó que la planificación familiar era un derecho de las parejas y las familias. Luego fue un asunto

2 La práctica de esterilización forzada en Bolivia era denunciada, por ejemplo, a través de films alternativos, como "Sangre de Cóndores". 
Los derechos (no) reproductivos en Argentina

central en la Conferencia Internacional de Población llevada a cabo en Bucarest en 1974, en la que volvió a resguardarse este derecho de las parejas y los individuos de decidir la cantidad y el momento para tener o no hijos(as). Más tarde en 1979, fue incorporado en la Convención sobre Eliminación de toda Forma de Discriminación contra las Mujeres, como un derecho básico de las féminas. En este documento los derechos reproductivos de las mujeres son reconocidos en varios artículos, subrayando asimismo, el derecho a tener acceso a información, educación y los medios que permitan el ejercicio efectivo de los mismos. ${ }^{3}$

\section{El movimiento por la Salud de las Mujeres}

A pesar de estos acuerdos internacionales, seguían produciéndose, sobre todo en los países del Tercer Mundo, violaciones a los derechos de planificación familiar (tal como se los llamaba entonces) que afectaban principalmente a las mujeres, a quienes se les indicaban métodos sin información adecuada y suficiente, $\mathrm{o}$ directamente se practicaban esterilizaciones forzadas. Muchas veces la recomendación o el uso de un método anticonceptivo se realizaba sin tener en cuenta el estado general de salud de las mujeres, es decir, sin considerar factores de riesgo como la hipertensión arterial, por ejemplo, o los efectos colaterales previstos por la contracepción química o mecánica.

Estos problemas dieron origen a que, junto con la efervescencia del movimiento de mujeres, empezara a gestarse en estos países un movimiento por los derechos y la salud reproductiva de las mujeres. ${ }^{4}$ En este caso, derechos reproductivos

3 NNUU, Convención Contra Toda Forma de Discriminación contra la mujer, 1979.

4 Recordemos que desde los sesenta se estaba produciendo con fuerza un nuevo florecimiento del feminismo en lo que se llamó la segunda ola cuyas dos principales características son le hecho de dejar de ser un movimiento de mujeres excepcionales y de poner al cuerpo y la sexualidad en el centro del debate público-político. 
significaba, principalmente, tener control sobre los métodos anticonceptivos para que éstos no fueran aplicados coercitivamente a las mujeres. Producto de todo este movimiento es que a principios de los ochenta, a raíz de una reunión internacional de mujeres realizada en Amsterdam se crea la Red Mundial por los Derechos Reproductivos (http://www.wgnrr.org). Pero al mismo tiempo, existió todo otro movimiento paralelo, preocupado por la salud de las mujeres, sobre todo por la salud reproductiva de las mujeres, proceso a partir del cual, en 1984 se funda en Colombia La Red de Salud de las Mujeres de América Latina y el Caribe (de Baribieri, 2000:50; www.reddesalud.org).

Nuevamente a mediados de los ochenta vuelve a la escena la alarma neomalthusiana, esta vez, con la justificación de la degradación del medio ambiente. El foco se puso, una vez más, sobre las mujeres de los países periféricos, como causantes de los problemas del mundo. Entonces es cuando las redes se conforman con fuerza y presionan ante la Organización Mundial de la Salud y otros organismos internacionales a favor de la salud y los derechos reproductivos de las mujeres. Desde entonces la denominación planificación familiar va cediendo paso ante la de "salud reproductiva" en los organismos internacionales después de que este concepto fuera introducido en las Redes de Salud y por los Derechos Reproductivos que mencionábamos anteriormente, aunque su origen se ha ido diluyendo al mismo tiempo que el carácter revulsivo que el término alguna vez portara.

Después de algunos debates, la Salud Reproductiva ha sido definida como

...un estado general de bienestar mental y social y no de mera ausencia de enfermedades y dolencias, en todos los aspectos relacionados con el sistema reproductivo y sus funciones y procesos. Esto entraña la capacidad de disfrutar de una vida sexual satisfactoria y sin riesgos y de procrear, y la libertad para decidir hacerlo o no hacerlo, cuando y con qué frecuencia. Esta última condición lleva implícito el derecho humano del hombre y la mujer a obtener 
Los derechos (no) reproductivos en Argentina

información sobre métodos para regular la fecundidad que no estén legalmente prohibidos, y acceso a métodos seguros y eficaces, asequibles y aceptables, y el derecho a recibir servicios adecuados de atención de la salud que permitan los embarazos y los partos sin riesgos y den a las parejas las máximas posibilidades de tener hijos sanos. ${ }^{5}$

La definición de salud reproductiva, cuyo supuesto era el ser punto de intersección entre las reivindicaciones por el aborto legal y contra las políticas antinatalistas coercitivas, incluida en las declaraciones de El Cairo y Beijing, no contempla sin embargo, el tema de la interrupción voluntaria del embarazo como uno de los aspectos de la salud reproductiva, siendo éste uno de los que generó mayores controversias en esas conferencias debido a la posición mantenida por el Vaticano, que se basa en la idea de "defensa del derecho a la vida desde el momento de la concepción".

\section{Los derechos (no)reproductivos desde Naciones Unidas al mundo. El Cairo y Beijing como puntos de inflexión}

Sin embargo, y aún con las restricciones mencionadas, esa noción de derecho/salud reproductiva anteriormente citada va a ser incorporada en el ámbito de Naciones Unidas a partir de dos Conferencias internaciones que se han constituido en hitos por los derechos/salud reproductiva de las mujeres en el ámbito internacional: El Cairo, 1994 y Beijing, 1995 (Scavone, 1999a). El Programa de Acción de El Cairo se propuso avanzar sobre la igualdad de género y el empoderamiento de las mujeres. E insistió sobre la salud como un derecho básico, de ahí que entre las propuestas de acción se encuentren la reducción de los índices de mortalidad materna y la necesidad de garantizar el acceso universal a la salud reproductiva. Así, marca un punto de clivaje, puesto que por primera vez, se incluye una definición explícita de

5 Plataforma de Acción, Beijing, 1995, párrafo 94. 
los derechos reproductivos dentro del marco de los derechos humanos, tomando a todos ellos como parte de una totalidad en estrecha relación unos con otros.

Beijing marcó un hito en la superación a la dicotomía planteada entre países centrales y países periféricos respecto de la consideración de los derechos (no)reproductivos y sexuales y su vinculación con las políticas de población. El tema del control de la natalidad fue planteado en forma independiente de las políticas de población y de los intereses geoestratégicos de los estados de modo claro y preciso. El control de la reproducción - los derechos (no)reproductivos y sexuales - fue reconocido como un derecho humano básico de las mujeres a decidir sobre sus propias vidas, su sexualidad y su fertilidad. Así, en Beijing reconoció que

Los derechos Humanos de la mujer incluyen el derecho a tener control y decidir responsablemente sobre los asuntos relativos a su sexualidad, incluidas su salud sexual y reproductiva, libres de coerción, discriminación y violencia. ${ }^{6}$

Para dar cumplimiento a esto instó a los gobiernos a tomar las medidas necesarias para garantizar estos derechos.

Es importante recordar aquí que el término derechos reproductivos no es un concepto impuesto por los organismos internacionales sino que tiene que ver con el movimiento de mujeres y las feministas Como señalamos más arriba, el movimiento de las mujeres por los derechos reproductivos tiene su punto de origen en las luchas concretas de las mujeres por lograr autonomía a partir del control de su cuerpo y el ejercicio de una sexualidad libre del mandato por una maternidad $y$ heterosexualidad obligatorias. Fueron ellas quienes presionaron por la incorporación de estos temas en el ámbito internacional a partir de la movilización, la presión y el lobby ejercido en las

${ }^{6}$ Plataforma de Acción de Beijing, parráfo 96. 
Los derechos (no) reproductivos en Argentina

Conferencias Internacionales y en las reuniones preparatorias (Sen, Germain y Chen, 1994). Es en estas dos Conferencias (El Cairo y Beijing) donde los conceptos de salud y derechos reproductivos se incorporan explícitamente en las Plataformas de Acción diseñadas, aunque su incorporación reconoce costos, pérdidas y algunas posibilidades producto de las relaciones de fuerza de una negociación compleja: inter e intra movimientos de mujeres y feministas norte - sur y, a su vez, de los movimientos con los distintos Estados nacionales y otros actores sociales relevantes, como la iglesia católica en latinoamerica, por lo menos.

\section{Los derechos (no)reproductivos como derechos ciudadanos. Algunas precisiones}

Dentro del consenso acordado, el concepto "Derechos Reproductivos" es entendido como un derecho humano básico que está relacionado con la libertad de las mujeres y sus parejas de elegir y decidir acerca de si tener hijos o no y cuándo. En este sentido,

...implican la posibilidad de ejercer la sexualidad y la maternidad en libertad, sin riesgos para la salud y sin ser objeto de discriminación cultural, social y laboral. Por lo tanto, incluyen la posibilidad de tomar decisiones reproductivas basadas en información veraz y accesible, y en recibir la protección social necesaria para llevar adelante estas decisiones. Para ello debe asegurarse el acceso a: anticoncepción segura y eficaz, atención y protección del embarazo y la maternidad, aborto seguro y legal, tratamientos para la esterilidad, servicios de prevención y tratamiento de enfermedades de transmisión sexual. Este acceso debe garantizar la protección médica y jurídico legal contra la violencia y los abusos sexuales, médicos, tecnológicos en materia de salud reproductiva (AAVV, 1997:149). 
Según Martha Rosenberg, psicoanalista, feminista y fundadora del Foro por los Derechos Reproductivos, este término "Aparece en los 90 - con toda una movida internacional de Salud de la Mujer - ...[el término]... tiene un poco del movimiento internacional de la mujer y tiene mucho de garantía". ${ }^{7} \mathrm{La}$ introducción específica del término derechos reproductivos al interior del movimiento de mujeres y las feministas en Argentina, tiene que ver con dos órdenes de factores, asociados a su vez al resurgimiento de la cuestión ciudadana y al paradigma de los derechos humanos. Al decir de Rosenberg, el tema adquiere fuerza ante las políticas sociales y económicas desarrolladas por el menemismo, que al tono de un cierto consenso neoliberal, producen un ensachamiento de la brecha entre ricos y pobres y un aumento exponencial de las desigualdades sociales.

Una batería de políticas implementadas por el menemismo produjo como consecuencia que amplios márgenes de personas quedaran fuera de la cobertura de servicios básicos $e$ indispensables para pertenecer a una comunidad de iguales. ${ }^{8}$ Salud, educación, empleo y garantías previsionales, y en muchos casos vivienda y alimentación, fueron derechos ciudadanos básicos de los que se vieron desprovistos una creciente cantidad de mujeres y varones durante esa etapa en la que, paradójicamente, se pregonaba el ingreso de la Argentina al primer mundo. En el marco de ese proceso de desciudadanización real en función del incremento exponencial de las desigualdades sociales, reclamar por derechos (no)reproductivos suponía apuntar en un movimiento en pinza hacia los dos contrapuntos que Fraser (2002) ha señalado en más de una oportunidad: reconocimiento y distribución en la puja por derechos ciudadanos que tensan la noción clásica de ciudadanía, tal es el caso de los (no)reproductivos.

7 Comunicación personal, Buenos Aires, agosto de 2000.

8 Menemismo refiere al sector del partido justicialista liderado por Carlos Menem quien ocupara el sillón presidencial durante 1989-1999. 
Los derechos (no) reproductivos en Argentina

\section{Los derechos (no)reproductivos como punto de convergencia}

Pero no sólo el contexto de resurgimiento del debate en torno de la condición ciudadana fue lo que operó como factor determinante en relación con un deslizamiento de la demanda por legalización del aborto a la reinvidicación por derechos (no)reproductivos. ${ }^{9}$ En cuanto a los otros factores influyentes o concurrentes, la mencionada psicoanalista afirma que, la denominación "Derechos Reproductivos", es producto de la convergencia de dos grandes puntos de tensión.

La génesis de la idea de derechos reproductivos se halla, por un lado, en la lucha feminista por el aborto llevada a cabo los países centrales en las décadas de los sesenta y setentas, asociadas con el control del propio cuerpo, de la fertilidad, de la capacidad reproductiva de las mujeres. Es decir, con las luchas por el aborto libre y gratuito en Inglaterra, Italia, Francia, EEUU. Pero, por otro lado, tiene que ver con las políticas de población coercitivas impuestas a las mujeres de los países del tercer mundo, $e$ incluso a minorías de los países del norte, como las mujeres negras, por ejemplo. En la India, particularmente, pero en Asia en general, en donde se aplicaron políticas poblacionales antinatalistas sumamente coercitivas, existe un movimiento muy fuerte por los derechos reproductivos. En ese contexto, como lo señalábamos anteriormente, derechos reproductivos significa

"Queremos tener control de los métodos anticonceptivos", puesto que no se intentaba que las mujeres se apropiaran

9 La crisis de los Estados de Bienestar y el desmoramiento de muchas de las políticas sociales que permitían asegurar cierta igualdad social y bienestar de las sociedades junto con el auge del discurso y las políticas neoliberales pusieron nuevamente en cuestión el tema de la ciudadanía, los derechos, la democracia. Dos textos claves de ese resurgimiento de los debates ciudadanos son: Marshall y Bottomore,1998 [1949] y el no 7 de la Revista Agora, dedicado exclusivamente a la temática con artículos de Kymlicka y Norman, Jelin, Held, etcetera. 
del derecho a decidir sobre su cuerpo sino que "se compraba la fertilidad de las mujeres. ${ }^{10}$

Para dar cabida a estos dos puntos conflictivos es que nace el término "Derechos Reproductivos", que luego se logra introducir en las resoluciones de los organismos internacionales y de este modo, se legitima y le da fuerza al movimiento de mujeres para llevar a cabo reivindicaciones e impulsar acciones en ese sentido en los distintos países.

\section{Dificultades teóricas y políticas de la noción "derechos sexuales y reproductivos"}

He realizado un recorrido histórico tratando de seguir la genealogía del concepto en función del cual se demandan "derechos (no)reproductivos y sexuales", "derechos reproductivos" $y / 0$ "salud sexual $y$ reproductiva" fundamentalmente en nombre de las mujeres. Muchas veces se dice que estos derechos son para todos y para todas, incluso para quienes no son heterosexuales o quienes no se incluirían ni como varones ni como mujeres: travestis, transexuales, transgéneros, intersexs. Sin embargo, la denominación y el sujeto colectivo que lleva adelante las demandas de cara al Estado, en este caso el Argentino, así como la traducción de las conquistas en términos de leyes $e$ incluso de políticas públicas, señala a las mujeres heterosexuales adultas como las principales y, en muchos casos, las únicas interlocutoras válidas en torno de estos asuntos.

De lo dicho hasta aquí surgen dos nudos críticos que han sido señalados por teóricos, teóricas y militantes, tanto feministas como pertenecientes al movimiento de mujeres. ${ }^{11}$ Estos anudamientos tensos son: el acento en la reproducción y los sujetos a los que interpela: finalmente, a las mujeres en tanto madres. En pocas palabras: el orden legal, pero sobre todo el de

${ }^{10}$ Martha Rosenberg, comunicación personal, agosto del 2001.

${ }^{11}$ Miler, 2000:68-109 y 2003; Pecheny, 2004; Raupp Ríos, 2004:164-185. 
Los derechos (no) reproductivos en Argentina

las políticas públicas concretas que mediatizan esa ley o leyes (la nacional o las provinciales), incluyen principalmente a mujeres adultas, heterosexuales, preferentemente con parejas, en edad de procrear. Y las políticas ofrecidas ponen el acento en la variable reproductiva de la denominación, lo que supone un énfasis en políticas de seguridad relativas a la prevención de ITS, controles génito - mamarios y atención antes, durante y después del embarazo. Considera en general la anticoncepción, pero siempre vinculado a lo anterior, en términos de profilaxis o como cuidado de la salud de las mujeres. Los varones, las personas no heterosexuales, los niños y las niñas y los y las adolescentes, los y las viejos y viejas tienen muchas más dificultades de ser considerados en el marco de estas políticas.

Si la demanda por derechos (no)reproductivos estaba ligada, como decíamos, al reclamo sesentista de libertad, autonomía y libre ejercicio de la sexualidad, lo ocurrido con el concepto y sus interpretaciones a través del tiempo señala algunos límites aunque también apertura de posibilidades, que es preciso señalar.

Retomando el inicio y esquematizando podríamos decir que el término derechos (no)reproductivos y sexuales incluye tres aspectos: 1) la seguridad en la reproducción, y los eventos relacionados con ello; 2) anticoncepción y aborto, los aspectos de la opción no reproductiva; 3) el libre ejercicio de la sexualidad. El consenso ha sido puesto primordialmente sobre la reproducción aún cuando se incluyan algunas posibilidades de anticoncepción. El punto de acuerdo en torno de salud reproductiva excluye la discusión sobre el tema del aborto y el ejercicio de sexualidades no heteronormativas - reproductivas.

En efecto, en otro trabajo había señalado que la imposibilidad de debatir la cuestión del aborto en el terreno político provenía del imperativo de separación entre sexualidad y reproducción que el reclamo asumía (Brown, 2001). No se trata sólo de tomar al aborto y su legalización como uno de los asuntos más complejos y controvertidos en todas las sociedades; o de 
adentrarse en el debate bioético que podría desplegarse a su alrededor. Se trata, precisamente, de que, en contraposición con el imperativo que pone en equivalencia los significantes madre, mujer y esposa, el aborto supone una ruptura, un quiebre, una fisura; funciona incluso como un gesto de resistencia a aceptar las definiciones hegemónicas de mujeres vigentes en nuestras sociedades (Rosenberg, 1998). Así, siguiendo esta línea, la imposibilidad de debatir públicamente de aborto, en una sociedad como la Argentina, es coherente con la enorme dificultad de introducir en el discurso público y mucho más en el institucional, imágenes de ser mujeres diferentes a la tradicional, de poner en circulación la idea de la no - maternidad y del ejercicio de sexualidades no hetero - normativas (Brown, 2001; Belluci, 1994).

Rápidamente se advierte cómo la idea de derechos (no)reproductivos y sexuales, al menos la que tenía un grupo de feministas y del movimiento de las mujeres, contrasta con el modo en que pudo insertarse en Naciones y Unidas y luego, en las respectivas legislaciones nacionales. En el caso Argentino, la transformación de la demanda por derechos reproductivos o derechos sexuales y reproductivos en algunos casos, apoyada en el consenso logrado en Naciones Unidas (que ya había excluido del debate asuntos relativos al aborto y a las sexualidades no heteronormativas), devino en Ley Nacional de salud sexual y procreación responsable en el año 2002. En las distintas provincias las denominaciones varían pero se mantienen dentro de lo que consideramos el consenso básico en torno de la salud reproductiva, tal como señalé anteriormente.

Como he adelantado en algunos trabajos previos (Brown, 2001, 2004b), esa denominación y esa conquista tanto en terreno legal como en el de las políticas públicas tiene sus pro y sus contras. Por un lado, significa haber podido hacer ingresar en el orden del derecho demandas y necesidades relegadas $e$ invisibilizadas por largo tiempo, tales como los derechos reproductivos, derechos bisagra entre lo público y lo privado y que ponen en evidencia el carácter sexuado de los sujetos y las sujetas, 
Los derechos (no) reproductivos en Argentina

al tiempo que tensan la noción clásica de ciudadanía cuya igualdad se edificaba sobre la base de una neutralidad $y$ universalidad de un modelo de sujeto, que lejos de cualquier abstracción, aparece marcado como varón, adulto y heterosexual. Por otro lado, una ley, aún en esos términos restrictivos, que garantice algunos servicios de salud reproductiva significa la creación de deberes y derechos, tanto por parte del Estado como de los ciudadanos y las ciudadanas a quienes interpela como tales, toda vez que lo que oficia de mediación es una ley; y un ciudadano o una ciudadana es por definición un sujeto o una sujeta portadora de derechos. Los derechos ciudadanos suponen obligaciones y garantías, pueden por lo tanto, ser reclamados y demandados en todas sus instancias, incluso la judicial.

En relación con la cuestión importantísima de que una demanda, particularmente aquellas provenientes de los sujetos y las sujetas subalternos y subalternas, se incorpore en términos legales, tiene una serie de implicancias que trascienden lo meramente legal impactando en lo subjetivo y lo político.

En términos políticos implica que esa demanda ha podido ingresar en el debate público - político y aún más en el institucional. Que un asunto sea discutido públicamente implica que ha logrado adquirir el estatuto de un tema relevante y se ha transformado en una cuestión de interés general trascendiendo el estigma de particularismo, intimidad y privacidad a la que suelen asociarse los temas vinculados con la reproducción, la anticoncepción, el aborto y las sexualidades.

La inscripción en el terreno legal supone en los regímenes políticos modernos, reconocimiento. Recordemos que,

Sólo como sujeto de derecho un individuo es reconocido en el espacio público como investido de una cierta potestad. Sólo como sujeto de derecho un sujeto es existente para el cuerpo social, y sus actos son sancionados con la fuerza de la ley que instaura lo permitido y lo prohibido, pero que a la vez lo faculta para la realización de una inmensa gama de 
Josefina Leonor Brown

acciones... quien no entre en el orden de la ley está por fuera del orden humano (Ciriza, 1996/7).

Pero además, el discurso del derecho tiene, al decir de Segato, una enorme eficacia simbólica.

Desde la perspectiva de los minorizados, el discurso del derecho, entendido como un eficaz sistema de nombres en permanente expansión tiene el poder de agitación, el carácter de propaganda, aún apuntando en la dirección de lo que todavía no existe, que aún no es posible adquirir en la vida social (Segato, 2003:127).

Sin embargo, esto último constituye para el caso de la salud reproductiva un juego de doble filo. Por un lado, tiene esas bondades señaladas en tanto adquiere estatuto legal pero, por otro, el desplazamiento al campo de la salud supone ir hacia la tendencia inercial del Estado que en el mismo movimiento que otorga derechos universales transforma algunas diferencias (de clase y sexo/género, de raza) en desigualdades. En este caso, el desplazamiento desde la noción "derechos (no)rerpoductivos y sexuales" concebidos como derechos civiles, hacia otra: la de "salud reproductiva" pone en riesgo uno de los desafíos de la demanda, cual es politizar y discutir en términos públicos y políticos asuntos relativos a los cuerpos, las sexualidades y la reproducción, históricamente relegados a un terreno personal, íntimo y privado y por ende no relevante en el orden de lo político. La institucionalización de los derechos (no)reproductivos $y$ sexuales en términos de salud reproductiva despolitiza $y$ medicaliza el reclamo, en el sentido de que éste deviene nuevamente algo que se dirime en el ámbito privado de un consultorio bajo el saber experto de los(as) profesionales de la salud. ${ }^{12}$

${ }^{12}$ Para más precisiones, cfr Brown, 2005; Segato, 2003; Bidegain, 1995. 
Los derechos (no) reproductivos en Argentina

Finalmente, y para cerrar con la apertura de posibilidades (y algunos límites) que han venido de la mano de la conquista de la salud reproductiva en Argentina, también se relaciona con la posibilidad de que ser ciudadana plena y gozar de ciertos derechos reproductivos deje de ser una cuestión de privilegios que se distribuyen en el mercado y se constituya en un derecho ciudadano. ${ }^{13}$ Coincidimos en este sentido con Yuval Davis, cuando resalta que los derechos reproductivos, como cualquier otro, entendidos como "libertades" u "opciones" privadas, carecen de significado, especialmente para los/as más pobres sin un conjunto de condiciones mínimas. Es decir: no basta con que estén legislados, hacen falta políticas públicas eficaces que los garanticen (Yuval-Davis, 1997:16).

Es necesario entonces llamar la atención sobre el asunto de la legalidad y juridicidad. Para las mujeres así como para otros y otras diferentes, inscribir las demandas en el marco de la ley no es un tema menor, por cuanto ese es el espacio en el que adquieren visibilidad y reconocimiento como asuntos de orden público y objeto de demandas exigibles al Estado. Y la ciudadanía implica algo más que el "derecho a tener derechos", supone también las condiciones que hagan posible el ejercicio de esos derechos. Sin embargo, en la Argentina la cuestión jurídica sigue pesando notablemente como una marca de un pasado autoritario que hace que se vea en el derecho una cuestión fundamental, sobre todo para los/as más débiles. Pero no todo derecho legislado equivale a un derecho consagrado. Y, tampoco toda demanda es pasible de ni es deseable que, pueda ser traducida al lenguaje del derecho. El lenguaje de la ley al mismo tiempo que reconoce, generaliza y universaliza creando obligaciones y garantías, restringe, constriñe y limita.

\footnotetext{
${ }^{13}$ Es preciso recordar la indisoluble vinculación entre derechos civiles, políticos, culturales y sociales. Sin los últimos, los primeros carecen de sentido para amplias mayorías en Argentina.
} 
Sin embargo, la mayor virtud de la ley es que pone límites al ejercicio arbitrario del poder (en cualquier ámbito). De allí también, la importancia que tiene el ejercicio efectivo de la justicia, que se "...constituye en la última frontera donde los ciudadanos perciben si sus derechos son efectivamente respetados" (Méndez, 2000:4). Y también recordar, al mismo tiempo, que la justicia no es precisamente un lugar libre de coerción, ni de determinantes políticos, culturales, económicos, etcétera. Mucho menos neutral en términos de género y de clase, todo lo cual conspira para que los derechos de las mujeres y los y las sujetos y sujetas diferentes y subalternos - as vean realizados sus derechos y ejercida su ciudadanía plena en una sociedad compleja y plural. Quizás por ello sea el lugar que las mujeres intentan evitar mientras suele ser el lugar al que los sectores más conservadores de la sociedad argentina, como las asociaciones pro-vida, recurren cuando agotan otros recursos (Birgin, 2000; Brown, 2004a).

Desde que el tema de los derechos (no)reproductivos y sexuales ingresa al debate público y político en el panorama liberalizador que respecto de los cuerpos y las sexualidades se produjo con el renacer democrático en 1983 pero más notablemente en los noventa, el asunto ha sido objeto de intensas disputas. Mientras los movimientos de mujeres, feministas y LGTTBI han presionado por el reconocimiento de sus derechos, el otro actor relevante situado en una posición contraria ha sido la jerarquía católica argentina. Ambos actores políticos han ido variando sus tácticas y estrategias en función de obtener mayores consensos, alianzas y alcanzar sus objetivos. A fuerza de ser esquemática y siguiendo lo que afirmaba el antropólogo Norbert Elias es posible decir que los avances en términos de los derechos (no)reproductivos y sexuales pueden medirse por la fuerza y la violencia de la reacción del actor opositor en juego (Elias e Scotson, 2000). Si a inicios del debate, cuando las relaciones de fuerza eran bastante desvaforables al movimiento de mujeres y feministas, la jerarquía católica argentina realizaba algunas declaraciones 
Los derechos (no) reproductivos en Argentina

públicas generales, lobby y cabildeo con sus aliados y aliadas en las distintas esferas del Estado. Sin embargo, a medida que los movimientos de mujeres y feministas fueron conquistando algunos espacios y leyes las estrategias se fueron modificando así como intensificando algunas acciones de violencia. Desde 1995 una de los recursos usados por la jerarquía católica argentina fue actuar en el espacio público a partir de movilizaciones callejeras de sus feligreses o de alumnos y alumnas de colegios católicos. Entonces $\mathrm{y}$ de manera creciente se han multiplicado las organizaciones pro-vida que realizan publicidad, acciones en la vía pública, manifestaciones $e$ incluso forman a mujeres para participar de los encuentros anuales de mujeres (ENM). ${ }^{14}$ Muchas de estas intervenciones resultan en actos violentos como lo ocurrido en Mendoza en el año 2004 cuando el día previo a la realización de un ENM, la ciudad apareció empapelada con dibujos de fetos en los úteros con la leyenda "Mendoza en contra del aborto" y, el día de inicio de las actividades varios de los colegios donde se desarrollarían los talleres aparecieron pintados con diversas leyendas como "izquierdistas", "homosexuales", "aborteras", "asesinas". ${ }^{15}$ Sin embargo, cuando ninguna de estas acciones logra detener los avances legales, el último recurso es la justicia. Pedidos de inconstituconalidad de las leyes como ocurrió en el año 2002 cuando se puso en marcha el Programa Nacional de Salud Reproductiva y procreación responsable al que la jueza Garzón de Lascano diera curso oportunamente. Posteriormente se planteó un recurso de amparo de una familia para que a sus miembros no se les aplicara la ley Nacional (www.bioetica.org).

\footnotetext{
${ }^{14}$ He desarrollado esos temas con más detenimiento en Brown, 2003, 2004a, 2006a:131-156, 2006b.

${ }^{15}$ Tarducci y Tagliafiero, 2004; Tarducci, 2005; AAVV, Revista Brujas, Publicación feminista, año 23, nº 30, octubre del 2004.
} 
Josefina Leonor Brown

\section{Consideraciones finales}

En síntesis, la noción de derechos reproductivos, derechos sexuales y reproductivos, derechos (no)reproductivos y sexuales como hemos dado en llamarles, salud sexual y reproductiva, salud reproductiva, salud sexual y procreación responsable, etcétera son nociones superpuestas, interconectadas, polisémicas y cuyo sentido y alcance, igual que el contenido de las leyes (cuáles sean los objetos de regulación, quiénes sean los o las titulares de esos derechos, cuál sea su alcance, etcétera) y las políticas sociales en sentido más clásico son siempre objeto de disputas y negociación en un campo político y discursivo. Lo importante aquí, es que los conceptos así como las políticas, tienen una trayectoria y son consecuencia de determinado contexto. Y que, los resultados son siempre paradójicos, ambivalentes, ambiguos. Es decir, si bien la noción de salud reproductiva es altamente problemática por las razones anteriormente expuestas, sin embargo suponen un mínimo exigible al Estado en términos de derechos históricamente negados a un conjunto de seres humanos (las mujeres) también históricamente vulnerabilizados. Y fueron conquistadas de ese modo no sólo porque probablemente algunos de los efectos no queridos no pudieron ser previstos previamente por el movimiento de mujeres y las feministas (que como grupo desaventajado tiende a amplificar su debilidad aún cuando ello no siempre se corresponda con la realidad (Petraci, 2004) sino también porque algunos de sus sectores no acuerdan con los derechos sexuales $y$ menos con la despenalización/legalización del aborto al menos como postura públicamente sostenida. ${ }^{16}$ Decía, fueron conquistas

${ }^{16}$ Aclaro lo de la postura pública por aquello del doble discurso que señalaba Bonnie Shepard hace unos años y porque en Argentina tenemos en el presidente que ocupó más años ese cargo en la última etapa democrática, un ejemplo paradigmático. Menem fue el primero en oponerse a la ley de divorcio y a la despenalización/legalización del aborto. Y sin embargo, en 1999 su ex-esposa Zulema Yoma asumía públicamente un aborto realizado en compañía y con el consentimiento del propio Menem que éste ni aceptó ni negó. Poco tiempo después obtendría el divorcio, gracias al cual pudo contraer matrimonio en 
Los derechos (no) reproductivos en Argentina

en esos términos porque, en buena medida ese era el consenso social conseguido en ese determinado contexto histórico y social que luego, sufrió modificaciones para alcanzar algún consenso en el juego de negociación con otros sectores sociales.

Por eso es importante seguir la trayectoria de los conceptos y de las luchas por su reivindicación, así como ir siguiendo la evolución de la institucionalización de esas demandas a través de las conquistas legales en cada caso logradas. Porque la reconstrucción de esa genealogía nos permite señalar cuáles son los obstáculos y los límites pero también las posibilidades y potencialidades, e imaginar términos y demandas más acordes a nuestro tiempo presente, al mismo tiempo que se advierte la dificultad de engarzar lo deseable con lo posible cuando se mira hacia atrás. Cuando se realiza esa mirada retrospectiva también se advierte eso que suele escamotearse cuando la memoria se diluye en un presente sin fin para decirlo con Jameson (1995), esto es que los derechos de los grupos subalternos siempre son fruto de una conquista y deben ser sostenidos constantemente pues nadie los ha garantizado para siempre. De retrocesos, de contra ofensivas, también nos habla esa memoria que a veces se olvida. Con eso en mente es posible preguntarse hoy cómo seguir, porque tenemos una línea histórica que podemos reconstruir y de la que podemos recuperar aprendizaje en experiencias. Desde ese pie en la memoria podemos preguntarnos si hoy la reinvindicación en términos de derechos reproductivos y sexuales no ha perdido el impulso revulsivo inicial, precisamente porque han mediado algunas conquistas y avances legales $e$ institucionales en su nombre y, ganaríamos más apostando a un debate teórico político que en términos de derechos sexuales y (no)reproductivos o, de la demanda por un derecho democráctico a la sexualidad como

segundas nupcias con la modelo chilena Cecilia Bolocco. Ese mismo año, 1999, Cristina Fernández Meijide (alianza) hasta entonces aliada de las feministasy el movimiento de mujeres en pro de la legalización/despenalización del aborto, en plena campaña electoral se negaba a hablar del asunto políticamente (Shepard, Bonnie, 2000:111-142). 
propone Raup Ríos (2004), no silencie ni el aborto, ni las sexualidades no heteronormativas - reproductivas. Es un desafío no exento de bemoles aunque sea una preocupación cada vez más recurrente en el terreno político social.

\section{Referências bibliográficas}

AAVV. Nuestros cuerpos, nuestras vidas: propuestas para la promoción de los Derechos Sexuales y reproductivos. Buenos Aires, Foro por los Derechos Reproductivos, 1997.

BELluCI, Mabel. Decidir sobre el propio cuerpo es un derecho ciudadano. Feminaria, año VII, n 13, Buenos Aires, sept. 1994.

BIDEGAIN, Ana María. Control Sexual y catolicismo. En Las mujeres en la historia de Colombia. Tomo II, Mujer y Sociedad, Consejería presidencial para la política social, presidencia de la República de Colombia, Grupo Norma, Vitral, 1995.

Birgin, Haydeé. (comp.) El derecho en el género y el Género en el derecho. Buenos Aires, Editorial Biblios, 2000.

BROWN, Josefina. De cuando lo privado se hace público, o de cómo se construyen las políticas sobre sexualidad y (no)reproducción. El caso de Mendoza. En PeTRACCI, Mónica y RAMOS, Silvina. (comp.) La política pública de salud y derechos sexuales y reproductivos en la Argentina: un aporte para comprender su historia. Buenos Aires, CEDES/UNFPA, 2006a.

. Entre el silencio y el escándalo. El aborto como asunto de debate político en la Argentina. En WOLF, Cristina Scheibe; FAVERI, Marlene de; RAMOS, Tânia Regina O. (comps.) Seminario Internacional Fazendo Gênero 7 - Gênero e preconceitos, Florianópolis-SC, UFSC/UDESC, $2006 \mathrm{~b}$.

. Los derechos reproductivos en la encrucijada entre religión y política en Argentina. Trabajo monográfico presentado para la materia "Problemas de Género en América Latina", Maestría FLACSO, Buenos Aires, 2005.

- Derechos, ciudadanía y mujeres en Argentina. Revista Política y cultura, $\mathrm{n}^{\circ}$ 21, México, Universidad Autónoma Metropolitana, 2004a. 
Los derechos (no) reproductivos en Argentina

- Los debates legislativos en torno de los derechos reproductivos en Argentina. Actas VII Jornadas Nacionales de Historia de las Mujeres - II Congreso Iberoamericano de Estudios de Género, Comisión de la Mujer/GESNOA/UNSalta, 2004b.

. El impacto de la globalización en la ciudadanía de mujeres en Argentina. El caso de los derechos sexuales y reproductivos. Revista Confluencia Sociología, Mendoza, FCPyS, UNcuyo, 2003.

. Los derechos reproductivos como derechos ciudadanos. Debates, sociología, tesina de licenciatura, FCPyS, UNCuyo, 2001.

CIRIZA, Alejandra. Ciudadanía, neoliberalismo, democracia ¿En qué sentido se dice hoy ciudadanía de mujeres? Sobre las paradojas de la abstracción del cuerpo real. Seminario Internacional Ciudadanía y democracia en tiempos de globalización neoliberal, Bogotá, 3 a 5 de noviembre de 2004a.

et. alii. Dimensiones de la ciudadanía de mujeres. 19852001, Informe final proyecto de investigación. SECTyP. UNCuyo, 2004b.

. De contratos, derechos e individuos. Problemas y encrucijadas entorno a la condición ciudadana de las mujeres. El Rodaballo, año 3, n 5, Buenos Aires, 1996/7.

DE BARBIERI, Teresita. Derechos reproductivos y sexuales. Encruciadas en tiempos distintos. Revista Mexicana de Sociología, vol. 62, $\mathrm{n}^{\circ} 1$, México, Instituto de Investigaciones Sociales, ene-marzo 2000.

ELIAS, Norbert e SCOTSON, John. Os estabelecidos e os outsiders. Rio de Janeiro, Jorge Zahar Editor, 2000.

FRASER, Nancy. Política feminista en la era del reconocimiento. Una aproximación bidimensional a la justicia de género. Documento especialmente elaborado para Seminario PRIGEPP-FLACSO, Buenos Aires-Argentina, 2002.

GuTIÉRREZ, Ma. Alicia y DURAND, Teresa. Tras las Huellas de un porvenir incierto. En AAVV, Avances en la Investigación Social en Salud Reproductiva y Sexualidad. Buenos Aires, AEPA/CEDES/CENEP, 1998.

JAMESON, Frederic. El posmodernismo o la lógica cultural del capitalismo avanzado. Buenos Aires-Argentina, Paidós, 1995 [1991]. 
Josefina Leonor Brown

Marshall, Thomas y BotTomore, Tom. Ciudadanía y clase social. Madrid, Alianza. 1998 [1949].

MÉNDEZ, Juan. El Acceso A La Justicia, Un Enfoque Desde Los Derechos Humanos. En THOMPSON, José. (coord.) Acceso a la justicia y la equidad: estudio en siete países de América Latina. Costa Rica, Banco Interamericano de Desarrollo/Instituto Interamericano de Derechos Humanos, 2000.

MILER, Alice. La demanda por los derechos sexuales. III Seminario Regional, Derechos Sexuales, Derechos reproductivos, Derechos Humanos, CLADEM, 2003.

. Sexual but not reproductive. Exploring the junction and Disjunction of sexual and reproductive rights. Health and human Rights, vol. 4, n², 2000.

PECHENY, Mario. Lógicas de acción colectiva de los movimientos por los derechos sexuales: un análisis con aires abstractos de experiencias bien concretas. In: CÁCERES, Frasca y PECHENY, Terto. (eds.) Ciudadanía sexual en América Latina: Abriendo el debate. Lima, Universidad Peruana Cayetano Heredia/Ford Foundation, 2004.

Petraci, Mónica. Opinión, derechos y salud. Buenos Aires, Norma, 2004.

RAUPP Ríos, Roger. Notas para um direito da sexualidade democrático. En CÁCERES, Frasca y PECHENY, Terto. (eds.) Ciudadanía sexual en América Latina. Abriendo el Debate. Lima, Universidad Peruana Cayetano Heredia/Ford Fundation, 2004.

ROMERO, Mariana, RAMOS, Silvina et alii. Los médicos frente a la anticoncepción y el aborto. ¿Una transición ideológica? Buenos Aires, CEDES, 2001.

ROSEMBERG, Martha. Las mujeres como sujetos... De las elecciones reproductivas a las condiciones de las mismas y los derechos que las garantizan. La libertad de tomarlas. En AAVV, Nuestros cuerpos, nuestras vidas: propuestas para la promoción de los Derechos Sexuales y reproductivos. Buenos Aires, Foro por los Derechos Reproductivos, 1997.

SCAVONE, Lucila. Introducción. En SCAVONE, Lucila. (comp.) Género y Salud Reproductiva en América Latina. Caratago-Costa Rica, LUR Libro Universitario Regional, 1999a. 
Los derechos (no) reproductivos en Argentina

Anticoncepción, aborto y tecnologías conceptivas: entre la salud, la ética y los derechos. En SCAVONE, Lucila. (comp.) Género y Salud Reproductiva en América Latina. Caratago-Costa Rica, LUR Libro Universitario Regional, 1999b.

SEGATO, Rita. Las estructuras elementales de la violencia - Ensayos sobre género entre la antropología, el psicoanálisis y los derechos huamanos. Buenos Aires, Prometeo, 2003.

Sen, G.; Germain, A. y Chen, L.C. (eds.) Population Policies Reconsidered. Health, Empowerment and Rights. Boston-MA, Harvard Series on Population and International Health, 1994.

SHEPARD, Bonnie. The "Double discourse" on sexual and reproductve Rights in Latin America: The Chasm between Public policy and Private actions. Health and Human Rights, vol. 4, n 2, 2000.

TARDUCCI, Mónica. La Iglesia y los Encuentros Nacionales de Mujeres. Estudos Feministas, Vol. 13, nº 2, Florianópolis-SC, 2005.

y TAGLIAFIERO, Bárbara. Iglesia católica: Argentina ni diversa ni laica. Revista Política y cultura, $\mathrm{n}^{\circ}$ 21, México, Universidad Autónoma Metropolitana, 2004.

YuVAL-DAVIS, Nira. Mujeres, Ciudadanía y Diferencia. En HolA, Eugenia y Portugal, Ana María (eds.) La ciudadanía a debate. Ediciones de las mujeres, $\mathrm{n}^{\circ}$ 25, Santiago de Chile, Isis Internacional, 1997. 